Check for updates

Cite this: Phys. Chem. Chem. Phys. 2017, 19, 18774

\section{Correction: Experimental and theoretical investigations of infrared multiple photon dissociation spectra of glutamic acid complexes with $\mathrm{Zn}^{2+}$ and $\mathrm{Cd}^{2+}$}

\author{
Georgia C. Boles, ${ }^{a}$ Cameron J. Owen, ${ }^{a}$ Giel Berden, ${ }^{b}$ Jos Oomens ${ }^{\text {bc }}$ and \\ P. B. Armentrout (iD *a
}

DOI: $10.1039 / c 7 c p 90145 c$

Correction for 'Experimental and theoretical investigations of infrared multiple photon dissociation spectra of glutamic acid complexes with $\mathrm{Zn}^{2+}$ and $\mathrm{Cd}^{2+\prime}$ by Georgia C. Boles et al., Phys. Chem. Chem. Phys., 2017, 19, 12394-12406.

rsc.li/pccp

Although the overall conclusions of the original article remain unaffected (no experimental or theoretical IR spectra are changed, nor is any calculated thermochemistry at $0 \mathrm{~K}$ ), the thermal corrections to the Gibbs free energy at $298 \mathrm{~K}$ were mistakenly overestimated. Corrected $298 \mathrm{~K}$ free energies are given below in Tables 1 and 2 of the original article and Tables S1 and S2 given in the supplementary information. Note that only subtle changes are observed in the relative energies of $[\mathrm{Zn}(\mathrm{Glu}-\mathrm{H}) \mathrm{ACN}]^{+}$such that the relative order of all $298 \mathrm{~K}$ theoretically determined low-energy species remains unchanged. The $\mathrm{CdCl}^{+}(\mathrm{Glu})$ system also exhibits small changes in its relative $298 \mathrm{~K}$ energies, and the minimal consequence on the theoretical conclusions drawn in the text is outlined below. Because the changes are small, we have not corrected all references to relative energies in the text. Our conclusion that the B3LYP-GD3BJ and MP2(full) levels of theory are more consistent with experimental data still holds.

Page 12397, second paragraph, corrected: "B3LYP-GD3BJ and MP2(full) levels of theory predict the $\left[\mathrm{N}, \mathrm{CO}_{\mathrm{s}}, \mathrm{CO}\right]$-tgcggt conformer (Fig. 2) to be lowest in energy."

Page 12398, second paragraph, corrected: "A slight preference is observed for the $\left[\mathrm{CO}_{2}{ }^{-}\right]$-cgggtt conformer (Fig. 2), where this species lies 9-10 $\mathrm{kJ} \mathrm{mol}^{-1}$ above the ground conformer at the B3LYP-GD3BJ and MP2(full) levels of theory..."

Page 12402, fifth paragraph, corrected: "Here, an equilibrium distribution at $298 \mathrm{~K}$ of the five lowest-energy conformers, $\left[\mathrm{N}, \mathrm{CO}_{\mathrm{s}}, \mathrm{CO}\right]$-tgcggt, $\left[\mathrm{CO}_{2}{ }^{-}\right]-$cgggtt, $\left[\mathrm{CO}_{2 \mathrm{~s}}{ }^{-}\right]-c c g g g t,\left[\mathrm{~N}, \mathrm{CO}_{\mathrm{s}}, \mathrm{CO}\right]$-tggggt, and $[\mathrm{N}, \mathrm{CO}]$-tgtgtt, respectively, would have populations of about $22,68,7,2$, and $1 \%$ for B3LYP; 86, 2, 0.2, 11.5, and $0.2 \%$ for B3LYP-GD3BJ; 26, 63, 8, 3, and 1\% for B3P86; and 82, 3, 0.3, 15, and $0.1 \%$ for MP2(full). Therefore, the conclusion that $\left[\mathrm{N}, \mathrm{CO}_{\mathrm{s}}, \mathrm{CO}\right]$-tgcggt is formed experimentally is clearly appropriate, where contributions from $\left[\mathrm{N}, \mathrm{CO}_{\mathrm{s}}, \mathrm{CO}\right]$-tggggt are also likely given the analysis of the spectral comparison and theoretical population of the conformers $(2-15 \%)$. Across all levels of theory, the [N,CO]-tgtgtt conformer has a maximum population of about $1 \%$; thus, the probability that this conformer is greatly contributing to the measured spectrum (even though it reproduces the spectral features fairly well) is relatively low. Notably, the conclusion that the zwitterionic species are not significantly contributing to the experimental spectrum is consistent with the findings at the B3LYP-GD3BJ and MP2(full) levels of theory (2-3\% predicted population), but not B3LYP and B3P86 (71-75\% predicted population)."

\footnotetext{
${ }^{a}$ Department of Chemistry, University of Utah, 315 S. 1400 E. Rm. 2020, Salt Lake City, Utah 84112, USA. E-mail: armentrout@chem.utah.edu

${ }^{b}$ Radboud University, Institute for Molecules and Materials, FELIX Laboratory, Toernooiveld 7c, NL-6525 ED Nijmegen, The Netherlands

${ }^{c}$ van't Hoff Institute for Molecular Sciences, University of Amsterdam, Amsterdam, The Netherlands
} 
Table 1 Relative free energies (298 K) of $[\mathrm{Zn}(\mathrm{Glu}-\mathrm{H}) \mathrm{ACN}]^{+}$complexes $^{a}$

\begin{tabular}{|c|c|c|c|c|}
\hline Structure & B3LYP & B3LYP-GD3BJ & B3P86 & MP2(full) \\
\hline$\left[\mathrm{N}, \mathrm{CO}^{-}, \mathrm{CO}_{\mathrm{s}}\right]-g c g g t$ & 0.0 & 0.0 & 0.0 & 0.0 \\
\hline$\left[\mathrm{N}, \mathrm{CO}^{-}, \mathrm{CO}_{\mathrm{s}}\right]-g c g g c$ & 11.8 & 12.4 & 11.6 & 11.5 \\
\hline$\left[\mathrm{N}, \mathrm{CO}^{-}, \mathrm{CO}_{\mathrm{s}}\right]-g g g g t$ & 12.8 & 13.7 & 13.0 & 12.7 \\
\hline$\left[\mathrm{N}, \mathrm{CO}^{-}, \mathrm{CO}_{\mathrm{s}}\right]-g t g t c$ & 32.7 & 33.5 & 32.8 & 33.2 \\
\hline$\left[\mathrm{CO}_{2}{ }^{-}, \mathrm{CO}_{\mathrm{s}}\right]-g g g g t$ & 37.8 & 46.9 & 40.0 & 43.7 \\
\hline$\left[\mathrm{N}, \mathrm{CO}^{-}, \mathrm{CO}_{\mathrm{s}}\right]-g g g g c$ & 39.4 & 40.8 & 39.2 & 40.1 \\
\hline$\left[\mathrm{N}, \mathrm{CO}_{\mathrm{s}}{ }^{-}, \mathrm{CO}\right]-\operatorname{tgggt} t$ & 48.2 & 46.7 & 46.6 & 41.4 \\
\hline$\left[\mathrm{CO}_{2}{ }^{-}, \mathrm{CO}_{\mathrm{s}}\right]-g g g g c$ & 47.7 & 57.0 & 49.6 & 53.1 \\
\hline$\left[\mathrm{N}, \mathrm{CO}_{2 \mathrm{~s}}^{-}\right]-\operatorname{ttg} c g$ & 50.6 & 50.5 & 48.8 & 36.9 \\
\hline$\left[\mathrm{N}^{-}, \mathrm{CO}_{\mathrm{s}}, \mathrm{CO}\right]-t g_{-} g_{+} g_{+} g_{+} t$ & 57.0 & 61.6 & 56.5 & 57.9 \\
\hline$\left[\mathrm{N}, \mathrm{CO}^{-}, \mathrm{OH}_{\mathrm{s}}\right]-g g g g t$ & 52.2 & 49.0 & 55.1 & 45.5 \\
\hline$\left[\mathrm{N}^{-}, \mathrm{CO}_{\mathrm{s}}, \mathrm{CO}\right]-t g_{+} g_{-} g_{-} g_{-} t$ & 54.2 & 57.4 & 54.9 & 55.1 \\
\hline$\left[\mathrm{N}, \mathrm{CO}_{\mathrm{s}}{ }^{-}, \mathrm{CO}\right]-\operatorname{cgtg} c$ & 60.5 & 58.3 & 58.5 & 53.8 \\
\hline$\left[\mathrm{N}^{-}, \mathrm{CO}_{\mathrm{s}}, \mathrm{CO}\right]-c g_{-} g_{+} g_{+} g_{+} t$ & 65.9 & 69.2 & 65.1 & 66.4 \\
\hline$[\mathrm{N}, \mathrm{CO}]-g \operatorname{tg} t c$ & 60.4 & 69.2 & 63.5 & 72.8 \\
\hline$\left[\mathrm{N}^{-}, \mathrm{CO}_{\mathrm{s}}, \mathrm{CO}\right]-c g_{+} g_{-} g_{-} g_{-} t$ & 66.8 & 70.6 & 65.8 & 67.4 \\
\hline$\left[\mathrm{N}^{-}, \mathrm{CO}_{\mathrm{s}}, \mathrm{CO}\right]-\operatorname{tgggg} c$ & 68.7 & 71.6 & 69.5 & 69.6 \\
\hline$\left[\mathrm{N}^{-}, \mathrm{CO}_{\mathrm{s}}, \mathrm{CO}\right]-\operatorname{cggggg} c$ & 72.6 & 76.3 & 72.5 & 72.8 \\
\hline$\left[\mathrm{N}, \mathrm{CO}_{\mathrm{s}}\right]-\operatorname{tg} \operatorname{tgg}$ & 68.2 & 73.7 & 72.1 & 75.9 \\
\hline
\end{tabular}

Table 2 Relative free energies (298 K) of $\mathrm{CdCl}^{+}(\mathrm{Glu})$ complexes ${ }^{a}$

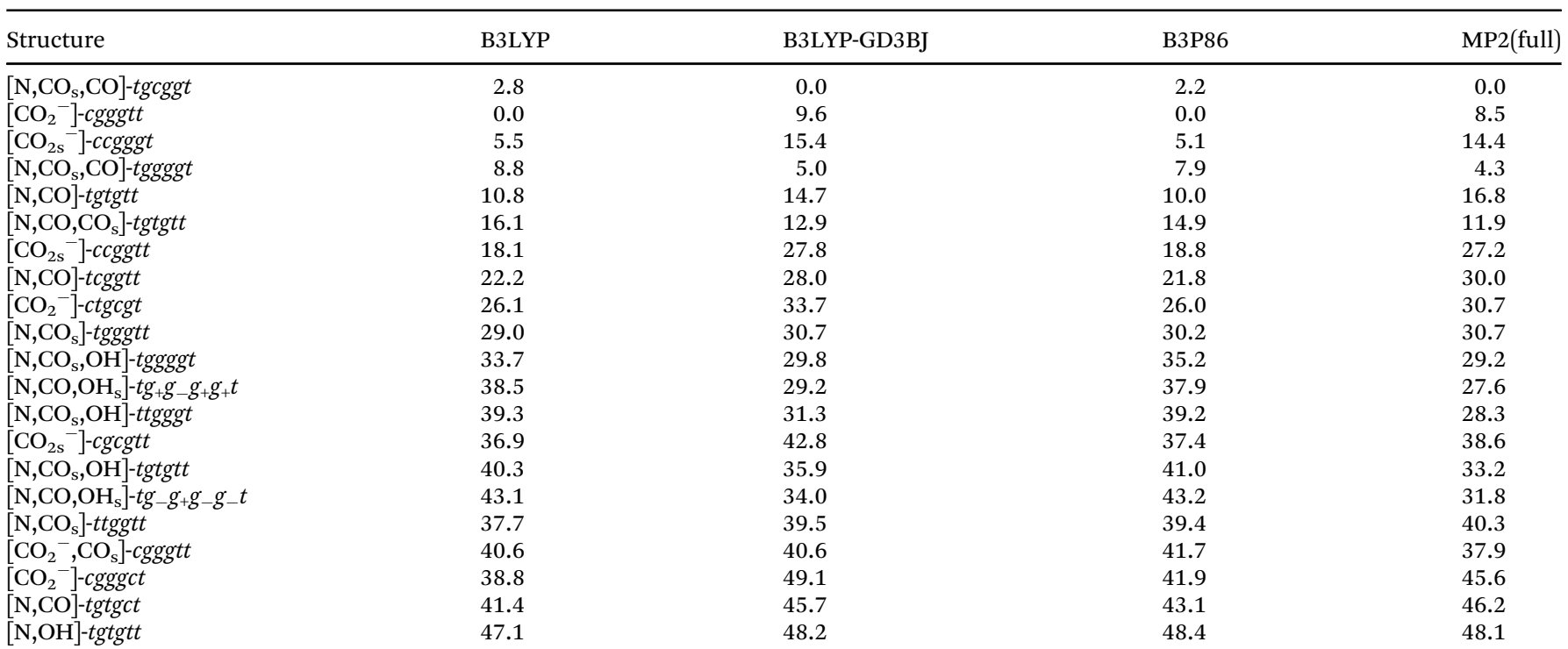

${ }^{a}$ Relative free energies calculated at the level of theory indicated using a def2-TZVPP basis set and SDD ECP for Cd. 
Table S1 Relative free energies (298 K) of higher energy $[\mathrm{Zn}(\mathrm{Glu}-\mathrm{H}) \mathrm{ACN}]^{+}$complexes $^{a}$

\begin{tabular}{|c|c|c|c|}
\hline Structure & B3LYP & B3P86 & MP2(full) \\
\hline$\left[\mathrm{N}^{-}, \mathrm{CO}_{\mathrm{s}}, \mathrm{CO}\right]-\operatorname{cgggg} c$ & 90.0 & 88.7 & 90.9 \\
\hline$\left[\mathrm{N}, \mathrm{C}_{\gamma}-\mathrm{CO}\right]-t \operatorname{tgggt} t$ & 80.7 & 78.5 & 85.4 \\
\hline$\left[\mathrm{C}_{\delta}{ }^{-}, \mathrm{CO}\right]-\operatorname{cgg} t t t$ & 83.3 & 81.9 & 94.4 \\
\hline$\left[\mathrm{N}, \mathrm{CO}_{\mathrm{s}}, \mathrm{CO}\right]-\operatorname{cggggg} c$ & 87.9 & 88.0 & 88.5 \\
\hline$\left[\mathrm{CO}_{2}{ }^{-}, \mathrm{OH}_{\mathrm{s}}\right]-g c g g t$ & 87.5 & 92.8 & 88.3 \\
\hline$\left[\mathrm{N}^{-}, \mathrm{CO}\right]-\operatorname{tg} t g t t$ & 85.5 & 90.1 & 99.9 \\
\hline$\left[\mathrm{N}^{-}, \mathrm{CO}_{\mathrm{s}}, \mathrm{CO}\right]-\operatorname{cgg} g g t$ & 95.3 & 95.2 & 95.5 \\
\hline$\left[\mathrm{N}^{-}, \mathrm{CO}, \mathrm{OH}_{\mathrm{s}}\right]-\operatorname{tgggg} t$ & 91.5 & 93.2 & 85.1 \\
\hline$\left[\mathrm{N}^{-}, \mathrm{CO}, \mathrm{OH}_{\mathrm{s}}\right]-\operatorname{tgggggt} t$ & 91.9 & 94.0 & 86.2 \\
\hline$\left[\mathrm{N}^{-}, \mathrm{CO}_{\mathrm{s}}, \mathrm{OH}\right]-$ tggggt & 93.3 & 97.4 & 92.8 \\
\hline$\left[\mathrm{N}^{-}, \mathrm{CO}_{\mathrm{s}}, \mathrm{OH}\right]-\operatorname{tgggggt} t$ & 96.1 & 100.0 & 94.6 \\
\hline$\left[\mathrm{N}, \mathrm{C}_{\gamma}{ }^{-}, \mathrm{CO}_{\mathrm{s}}\right]-\operatorname{tgggtc}$ & 93.3 & 90.7 & 97.4 \\
\hline$\left[\mathrm{N}, \mathrm{C}_{\gamma}-{ }^{-}, \mathrm{CO}_{\mathrm{s}}\right]-\operatorname{tgggtt}$ & 95.8 & 95.2 & 100.1 \\
\hline$\left[\mathrm{N}^{-}, \mathrm{CO}\right]-c g t g t t$ & 101.0 & 104.9 & 114.8 \\
\hline$\left[\mathrm{N}^{-}, \mathrm{CO}_{\mathrm{s}}\right]-c t c g t t$ & 109.0 & 110.6 & 118.5 \\
\hline$\left[\mathrm{N}^{-}, \mathrm{CO}, \mathrm{OH}_{\mathrm{s}}\right]-\operatorname{cgggggt} t$ & 107.1 & 108.1 & 100.2 \\
\hline$\left[\mathrm{N}^{-}, \mathrm{CO}_{\mathrm{s}}, \mathrm{OH}\right]-\operatorname{tgggg} c$ & 106.4 & 110.4 & 106.4 \\
\hline$\left[\mathrm{N}, \mathrm{C}_{\gamma}-\mathrm{CO}_{\mathrm{s}}\right]-\operatorname{cgtg} t t$ & 108.4 & 104.8 & 112.0 \\
\hline$\left[\mathrm{N}, \mathrm{CO}_{\mathrm{s}}\right]-\operatorname{cgtg} t$ & 105.2 & 107.6 & 114.4 \\
\hline$\left[\mathrm{N}^{-}, \mathrm{CO}\right]-\operatorname{tg} t g t c$ & 104.4 & 108.9 & 118.7 \\
\hline$\left[\mathrm{N}, \mathrm{C}_{\gamma}-, \mathrm{CO}_{\mathrm{s}}\right]-\operatorname{tgggtc}$ & 109.1 & 107.9 & 112.5 \\
\hline$\left[\mathrm{N}, \mathrm{C}_{\gamma}-{ }^{-}, \mathrm{CO}_{\mathrm{s}}, \mathrm{CO}\right]-\operatorname{cgtg} t t$ & 110.7 & 106.6 & 106.5 \\
\hline$\left[\mathrm{N}^{-}, \mathrm{CO}_{\mathrm{s}}\right]-\operatorname{ctcgtc}$ & 111.5 & 112.7 & 120.0 \\
\hline$\left[\mathrm{C}_{\gamma}{ }^{-}, \mathrm{CO}, \mathrm{COs}\right]-\operatorname{cggtg} c$ & 112.7 & 108.3 & 120.4 \\
\hline$\left[\mathrm{N}^{-}, \mathrm{CO}_{\mathrm{s}}\right]-\operatorname{cgtg} \operatorname{tg} c$ & 120.8 & 122.9 & 129.9 \\
\hline$\left[\mathrm{C}_{\gamma}{ }^{-}, \mathrm{CO}, \mathrm{CO}_{\mathrm{s}}\right]-\operatorname{cggggtc}$ & 122.8 & 118.8 & 126.8 \\
\hline$\left[\mathrm{N}^{-}, \mathrm{CO}\right]-\operatorname{cgtgtc}$ & 122.8 & 126.5 & 136.7 \\
\hline$\left[\mathrm{N}, \mathrm{C}_{\gamma}-\mathrm{CO}_{\mathrm{s}}, \mathrm{CO}\right]-\operatorname{cgtg} t c$ & 127.1 & 122.6 & 122.5 \\
\hline$\left[\mathrm{C}_{\beta}{ }^{-}, \mathrm{CO}_{\mathrm{s}}, \mathrm{CO}\right]-\operatorname{tggggtc}$ & 132.4 & 132.9 & 140.6 \\
\hline$\left[\mathrm{N}^{-}, \mathrm{OH}, \mathrm{OH}_{\mathrm{s}}\right]-\operatorname{tggggg} t$ & 139.1 & 144.6 & 129.0 \\
\hline$\left[\mathrm{N}^{-}, \mathrm{OH}\right]-t t t g t t$ & 141.1 & 149.0 & 151.4 \\
\hline$\left[\mathrm{N}, \mathrm{C}_{\gamma}{ }^{-}, \mathrm{CO}_{\mathrm{s}}\right]-\operatorname{cggggtc}$ & 151.8 & 150.7 & 156.4 \\
\hline$\left[\mathrm{N}^{-}, \mathrm{OH}\right]-t t t g t c$ & 154.7 & 162.5 & 164.9 \\
\hline
\end{tabular}

Table S2 Relative free energies (298 K) of higher energy $\mathrm{CdCl}^{+}(\mathrm{Glu})$ complexes $^{\mathrm{a}}$

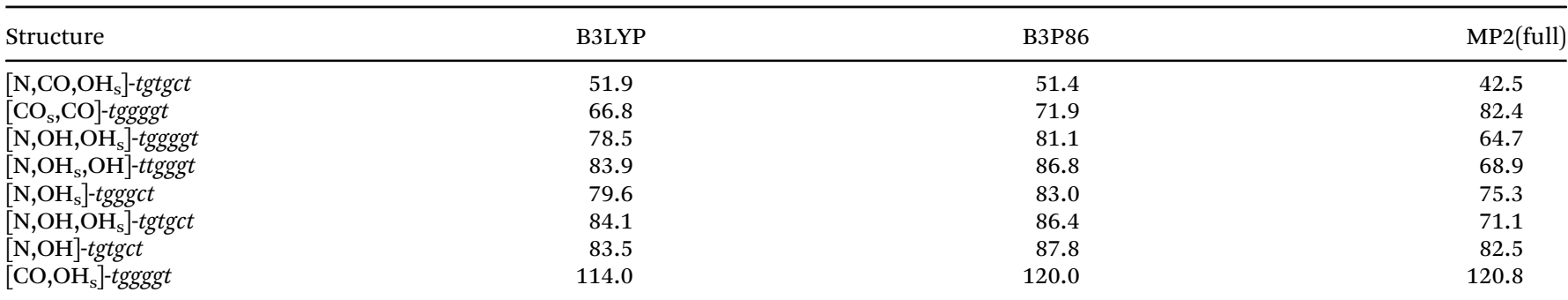

${ }^{a}$ Relative free energies calculated at the level of theory indicated using a def2-TZVPP basis set and SDD ECP for Cd.

The Royal Society of Chemistry apologises for these errors and any consequent inconvenience to authors and readers. 